\title{
The CFTR gene variants in Japanese children with idiopathic pancreatitis
}

\author{
Manami Iso ${ }^{1,2}$, Mitsuyoshi Suzuki', Kumiko Yanagi ${ }^{2}$, Kei Minowa', Yumiko Sakurai ${ }^{1}$, Satoshi Nakano ${ }^{1}$, Kazuhito Satou' \\ Toshiaki Shimizu ${ }^{1}$ and Tadashi Kaname $\mathbb{B}^{2}$
}

\begin{abstract}
The cystic fibrosis transmembrane conductance regulator (CFTR) gene has been reported as one of the pancreatitis susceptibility genes. Although many variants of CFTR have been reported in Caucasian patients, there are few data in Japanese patients. We aimed to survey CFTR variants in Japanese children with idiopathic pancreatitis. Twenty-eight Japanese paediatric patients with idiopathic pancreatitis were enroled, who were not previously diagnosed by genetic analysis of PRSS1 and SPINK1. The entire CFTR gene was sequenced in the patients by combining LA-PCR and nextgeneration sequencing analysis. To determine a splice-affecting variant, CFTR expression was investigated in the nasal epithelial cells by RT-PCR. One (3.6\%) and 15 (53.6\%) of 28 patients had pathogenic and functionally affected variants in the CFTR gene, respectively. Two variants, p.Arg352GIn and p.Arg1453Trp, were found more frequently in the patients compared with one in Japanese healthy controls ( $p=0.0078$ and 0.044 , respectively). We confirmed skipping of exon 10 in the nasal epithelial cells in one patient having a splice-affecting variant (c.1210-12T(5)) in intron 9. Functionally affected variants of the CFTR gene are not so rare in Japanese paediatric patients with idiopathic pancreatitis. Surveying CFTR gene variants in a Japanese sample could help identify pancreatitis risk in these children.
\end{abstract}

\section{Introduction}

There are many risk factors contributing to acute pancreatitis in children. Genetic analysis can be helpful in making the diagnosis of paediatric pancreatitis. The first report of a genetic cause of idiopathic pancreatitis demonstrated mutations in cationic trypsinogen $(P R S S 1)^{1}$. Since then, another causative gene for pancreatitis, serine protease inhibitor Kazal type 1 (SPINK1) ${ }^{2}$, and strongly associated genes, such as, chymotrypsinogen $(C T R C)^{3}$ and recently carboxypeptiase $\mathrm{A} 1(C P A 1)^{4}$, have also been reported.

The cystic fibrosis transmembrane conductance regulator (CFTR) gene has been identified as a causative gene for cystic fibrosis $(\mathrm{CF})^{5}$ and is also reported to be a gene associated with pancreatitis ${ }^{6,7}$. CFTR encodes a protein of

\footnotetext{
Correspondence: Tadashi Kaname (kaname-t@ncchd.go.jp) 'Department of Pediatrics and Adolescent Medicine, Juntendo University Graduate School of Medicine, 2-1-1 Hongo, Bunkyo-ku, Tokyo 113-8421, Japan 2Department of Genome Medicine, National Center for Child Health and Development, 2-10-1 Okura, Setagaya-ku, Tokyo 157-8535, Japan
}

1480 amino acid residues expressed in the apical membrane of exocrine epithelial cells and plays a role as a cAMP-dependent chloride channel ${ }^{5}$. To date, more than 2000 variants of the CFTR gene have been reported ${ }^{8,9}$.

Influences of CFTR function depend on its variants, which varies with symptoms or appearance of the disease. Many cohort studies examining the association between CFTR variants and pancreatitis have been conducted in western countries ${ }^{6,7,10,11}$. However, there are few reports on association between CFTR variants and idiopathic pancreatitis in Asian populations, except for a few studying alcoholic chronic pancreatitis ${ }^{12-16}$. Furthermore, there is no information about the genetic risk of CFTR variants in Japanese children with idiopathic pancreatitis. In our previous study, we performed genetic analysis of PRSS1, SPINK1, CTRC and CPA1, finding that 39\% (50/ 128) of paediatric Japanese patients with idiopathic pancreatitis had at least one pathogenic variant of those genes $^{17}$. However, there is no such data for the CFTR gene in this sample. Therefore, the aims of our study were to 
survey CFTR variants in Japanese children with idiopathic pancreatitis to determine any relationship between them.

\section{Methods}

\section{Subject and data}

This study was approved by the ethical review committee of Juntendo University (approval number 2017176) and National Center for Child Health and Development (approval number 1800). Written informed consent was obtained from each subject or their relatives before the study in accordance with the principles of the Declaration of Helsinki.

In total, 28 Japanese paediatric patients with idiopathic pancreatitis were analysed, who had no pathogenic variants of PRSS1 and SPINK1 by genetic analysis in a previous study ${ }^{17}$. In addition, their families and 92 healthy Japanese girls (9-12 years old) were enroled as healthy control subjects. In-house data of whole exome sequencing from randomly picked 1500 individuals, who were not related to pancreatitis, were also used as control.

\section{Targeted next-generation sequencing}

Genomic DNA was extracted from peripheral blood leucocytes. Sixteen primer pairs were designed to amplify the entire region of the CFTR gene (GenBank: NM_000492), spanning $\sim 200 \mathrm{~kb}$, including the promoter region, which is $2 \mathrm{~kb}$ upstream of the translation initiation codon of exon 1 (Supplementary Table S1). Longrange and accurate PCR (LA-PCR) was performed using KOD Multi \& Epi (TOYOBO, Co., Ltd, Osaka, Japan) under appropriate conditions listed in Supplementary Table S1. Each LA-PCR product was confirmed by agarose gel electrophoresis and the concentrations were measured on a Qubit fluorometer using dsDNA BroadRange assay kit (Thermo Fisher Scientific, Waltham, MA, USA). Then, the equal amount of amplicons were mixed and sheared to about $400 \mathrm{bp}$ fragments using Covaris S220 (Covaris, Inc., Woburn, MA, USA) in accordance to the manufacturer's instructions. The sheared products were then purified using Mini-Elute PCR Purification Kit (Qiagen, Hilden, Germany) and constructed libraries for GS Junior sequencer (Roche, Basel, Switzerland) with MID adaptors using GS Junior titanium Rapid library (shotgun) and emPCR (Lib-L) kits (Roche, Basel, Switzerland). Short fragments were removed using the AMPure beads kit (Agencourt, Beckman Coulter Genomics, Pasadena, CA, USA). Quality and product size were assessed on the 2000 TapeStation (Agilent Technologies, Inc., Santa Clara, CA, USA). Library quantification was determined by fluorometric measurements using a QuantiFluor ${ }^{\text {ris }}$-ST Fluorometer (Promega, Inc., Madison, WI, USA). Then, three libraries were mixed at equal quantity and amplified using the Lib-L emPCR Kit (Roche, Basel, Switzerland), following the manufacturer's instructions. Bead enrichment and sequencing were performed using GS Junior Titanium Sequencing Kit (Roche, Basel, Switzerland).

Sequence data processing, mapping and variant calling were assessed on the built-in software, GS Run Browser and GS Reference Mapper (Roche, Basel, Switzerland). The genomic data of GRCh37 and SNP135 were used as reference for variant calling. In addition, the reads were confirmed visually using the Integrative Genomics Viewer software $^{18}$ and all variants in the exons and some in the introns were confirmed by Sanger sequencing.

\section{Sanger sequencing}

In the next-generation sequencing (NGS) data, the detected variants and low coverage $(<8$ reads) of coding regions were validated by the Sanger sequence. Variants that were found in the patients were also sequenced in their families by Sanger sequencing as well; some family members were affected with pancreatitis. We also analysed sequencing data of splicing variants in intron 6 (GATT repeats) and 9 (poly $\mathrm{T}$ and TG repeats) from healthy Japanese girls as controls, as we had no information about the variant frequencies of these regions among the Japanese. Primers for the Sanger sequencing are listed in Supplementary Table S2. Sanger sequencing was performed using an ABI3130xl DNA Analyzer (Applied Biosystems, Foster, CA, USA).

\section{RNA analysis}

The nasal epithelial cells were collected by gently brushing the inferior turbinate using a cotton swab ${ }^{19}$. The swab was immediately immersed in a $350 \mu \mathrm{l}$ buffer RLT preservation solution (Qiagen, Hilden, Germany) and stored at $-20^{\circ} \mathrm{C}$. Total RNA was extracted using RNeasy Micro kit (Qiagen, Hilden, Germany) and reverse transcribed to cDNA using PrimeScript ${ }^{\text {tw }}$ RT reagent kit (Takara-Bio, Otsu, Japan).

The CFTR gene expression was investigated by reversetranscription quantitative PCR (RT-qPCR $)^{20}$. The primers and predicted sizes of the segments are shown in Fig. 1 and Supplementary Table S3. The glyceraldehyde-3phosphate dehydrogenase (GAPDH) gene was amplified and considered as control. Each PCR product was run through agarose gel electrophoresis and then validated by the Sanger sequence.

\section{Statistical analysis}

The variant frequencies in the Japanese population (except for splicing variants of intron 6 and 9) were obtained from in-house whole-exome sequencing data of 1500 non-pancreatitis controls or the Tohoku Medical Megabank Organization (ToMMo) 3.5k version 2 in the iJGVD ${ }^{21}$. The significance of the differences in variant frequencies between patients and controls was tested by 

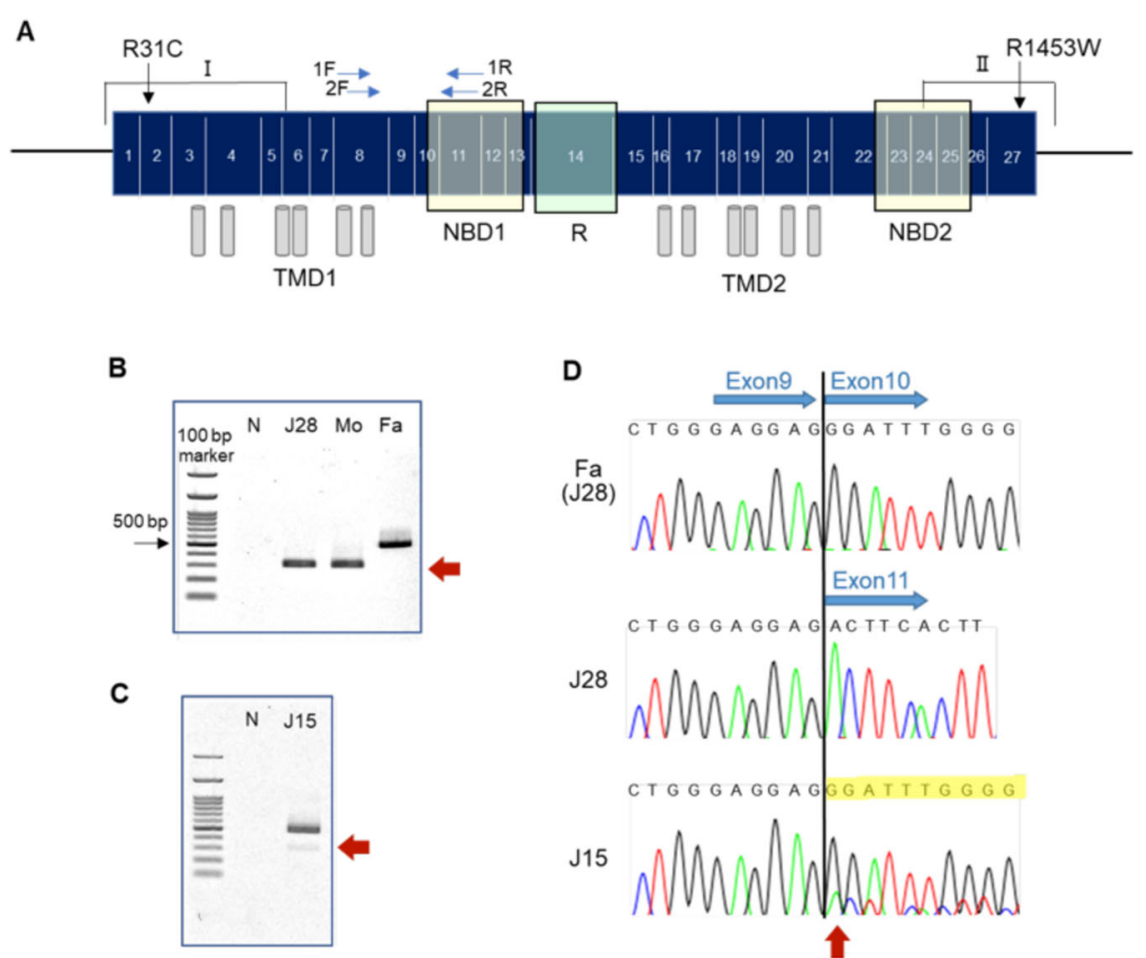

Fig. 1 Schematic representation of the CFTR CDNA and primer sites for RT-PCR, and skipping of exon 10. a Schematic diagram of the CFTR CDNA (exons) and domains, and amplified regions by RT-PCR. Nested-PCR primer sets to amplify exon 8-11 region were indicated as arrows (blue). NBD: nucleotide-binding domain, R: regulatory domain, TMD: transmembrane domain. b, c RT-PCR products of exon 8-11 in a patient J28 and his parents (b) and in $\mathrm{J15}$ (c) in the nasal epithelial cells. Fa: father of J28, J28: patient, Mo: mother of J28, N: negative control. The nested-PCR primer 2F and 2R gave $\sim 500$ bp fragments in the father and 300 bp fragments in the patient and mother. $\mathbf{d}$ Confirmation of the RT-PCR products of exon 8-11 by Sanger sequencing. The sequence revealed exon 10 skipping in J28 and his father (upper), and partial exon 10 skipping in J15 (arrow)

two-tailed Fisher's exact test, likelihood ratio test or $\chi^{2}$ test. A $p$-value of $<0.05$ was considered significant.

\section{Results}

\section{Genetic analysis}

Sequence analysis of the entire CFTR gene including promoter region, spanning about $200 \mathrm{~kb}$, was performed in 28 patients by NGS. The 20 most common CFTR mutations found in Caucasian populations were not found in these patients (E60X, R117H, R334W, R347P, A455E, $\Delta$ I507, $\Delta$ F508, G542X, G551D, R553X, c.489+ $1 \mathrm{G}>\mathrm{T}$ (previously noted as $621+1 \mathrm{G}>\mathrm{T}$ ), c.948del T (previously 1078delT), R1162X, S1251N, W1282X, N1303K, c.1585-1G > A (previously 1717-1G > A), c.2051_2052del insG (previously 2183AA $>$ G), c.3528delC (previously $3659 \mathrm{delC}$ ) and c.3718-2477C > $\mathrm{T}$ (previously $3849+10 \mathrm{kbC}>\mathrm{T})$ ).

We identified 8 non-synonymous variants, R31C, E217G, R352Q, V470M, I556V, L1156F, Q1352H and R1453W, and 1 synonymous variant, c.2562T $>$ G in 19 patients (Table 1). Of these variants, five are known to be unique to the Asian patients (E217G, I556V, L1156F, $\mathrm{Q} 1352 \mathrm{H}$, and $\mathrm{R} 1453 \mathrm{~W})^{12,14,16}$. Of the eight non- synonymous variants, R31C was recorded as uncertain significance in the most severe clinical significance or conflict in the latest status in the ClinVar database. V470M was recorded as likely benign in both statuses in the ClinVar database. Others were recorded as pathogenic/likely pathogenic or conflict, respectively (Table 1). Allele frequency of R352Q and R1453W were significantly higher in patients compared with one in the in-house control data $(p=0.018$ and 0.033 , respectively, Table 1$)$ and in the ToMMo_3.5K control population $(p=0.0078$ and 0.044 , respectively, Table 1$)^{21}$. The R352 variant is known as pathogenic and R1453W is known as pathogenic in most severe significance but as conflict in the ClinVar database. Of four patients with CFTR R1453W variant, one patient had a variant of p.A137G in CPA1, which was previously reported ${ }^{17}$ (data not shown). In this study, we could not find novel pathogenic variants in exonic region of CFTR in the patients.

A splice-affecting variant, 5_9T (c.1210-12 T(5_9)), in intron 9 was investigated in the patients and controls by Sanger sequencing (Table $1 \mathrm{~b}$ ). The heterozygous variant $5 \mathrm{~T}$ was identified in four patients, with an allele frequency of $7.14 \%$ (Table $1 \mathrm{~b}$ ). The allele frequency of $5 \mathrm{~T}$ was not 


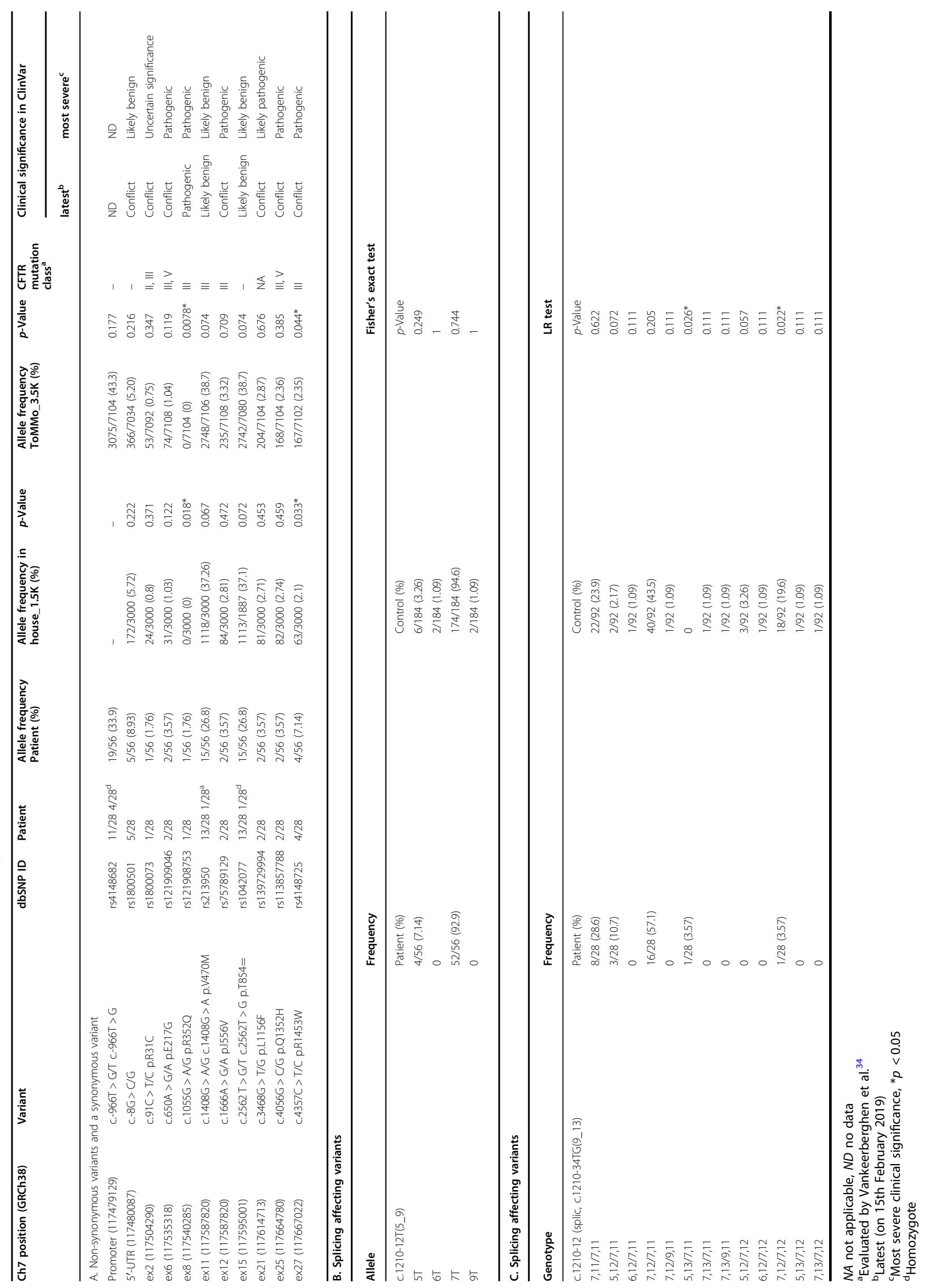


significantly different $(p=0.249)$ between patients and controls (Table $1 \mathrm{~b}$ ).

Many intronic variants were found in patients (Supplementary Tables S4 and S5) and some of these variants, such as rs370483286 (intron 1), rs371779267 (intron 3), rs547233512 (intron 6), rs138454021 (intron 10), rs180877927 (intron 11), rs112433140 (intron 15), rs139568843 (intron 17), rs183819332 (intron 18), rs147410641 (intron 20), rs7797932 (intron 21), rs1820871 (intron 10), rs4148706 (intron 10), rs143964990 (intron 10), rs34855237 (intron 10), rs869218449 (intron 11), rs535033297 (intron 18), rs213985 (intron 21) and rs371815480 ( $3^{\prime}$-untranslated region), were significant $(p<0.05$ and $p<0.01$, respectively) in the patients compared with Japanese ToMMo control population ${ }^{21}$ and 1000 Genome data of the East Asian population (Supplementary Table S4) ${ }^{22}$.

Genotype of TG repeats 11/13 (c.1210-34TG (11/13)), which repeats expansion might also affect splicing ${ }^{23}$, and 5T/7T (c.1210-12 T (5/7)), both in intron 9, was found in 1 patient but not in 92 normal controls (Table 1C and Table 2 (J28)).

For pathogenic variants, seven non-synonymous variants and one splice-affecting variant in intron 9 of CFTR, patients with variants and clinical feature were listed in Table 2 (five patients with V470M only are not listed). Of the 28 patients in this study, 16 patients (57.1\%) had nonsynonymous or splice-affected variant of CFTR (Table 2). There was no effect of sex on these findings. Onset of the disease ranged from 1 to 13 years. The main symptom of the patients was abdominal pain. The sweat chloride concentration test of patients, who had a result, was normal. There does not seem to be an obvious correlation between variant type and age of onset. In this study, patients with abnormal findings of endoscopic retrograde cholangiopancreatography and/or magnetic resonance cholangiopancreatography, such as stones and pancreatic duct dilatation, had a non-synonymous variant of R31C, E217G or Q1352H, which has been previously reported in chronic pancreatitis in adult patients ${ }^{14,16,24}$ (Table 2).

\section{RNA analysis}

Patient J28 had a genotype of (TG) $11 / 13$, (T) $5 / 7$ in intron 9, which might affect splicing of CFTR (Table 2). One allele of (TG)13, (T) 5 was inherited from his mother, who was affected with alcoholic-related pancreatitis. Results of RT-PCR for exon 8-11 of CFTR displayed a shorter fragment compared with control in the patient and the mother, but not in the father (Fig. 1b). Sanger sequencing of the RT-PCR product from the family revealed that exon 10 of CFTR was skipped in the nasal epithelial cells (Fig. 1d).

We analysed the CFTR gene expression in two patients, $\mathrm{J} 15$ and J28, with RT-qPCR ${ }^{20}$ and RT-PCR. J15 had a compound heterozygote of R1453W and R31C inherited from non-affected mother and father, respectively. CFTR expression was investigated in the nasal epithelial cells by RT-qPCR using primer pairs targeted for two regions (Fig. 1a; I and II). Gene expression and splicing pattern were not altered compared with normal control (data not shown).

We also analysed the splicing of CFTR in J15, who had genotype of (TG) 11/11, (T) 7/7 in intron 9 (Table 2). Partial skipping of exon 10 was observed in the patient (Fig. 1c, d).

\section{Discussion}

In this study, we investigated the entire CFTR gene in 28 Japanese paediatric patients with idiopathic pancreatitis using targeted NGS analysis and CFTR expression analysis. We found that 16 patients had non-synonymous or splice-affecting variant of CFTR out of 28 patients, who had no pathogenic variants of PRSS1 and SPINK1 by genetic analysis in our previous study ${ }^{17}$. We found eight non-synonymous variants of CFTR, R31C, E217G, R352Q, V470M, I556V, L1156F, Q1352H and R1453W, in the patients. R352Q was recorded as pathogenic in the ClinVar database (Table 1). The other variants, except V470M, are registered as conflict pathogenicity in the Clinvar database (Table 1). The pathogenicity of such variants was registered as based on CF-causing variants. In fact, the variants found in this study are known to affect function or expression of CFTR as described later, suggesting that those are functionally affected variants.

Up to now, CFTR analysis has been neglected in Japan as CFTR variants are thought to be rare in Japanese CF patients ${ }^{25,26}$. However, our findings imply that Japanese paediatric patients with idiopathic pancreatitis would have greater rates of CFTR variants than previously expected.

No patients in our study had any of the common CFcausing variants in Caucasians, consistent with other studies in Asian patients ${ }^{12-16,24}$. This suggests that Asian or Japanese populations may have different variants of CFTR than European populations. Of the eight nonsynonymous variants found in the patients, it appears that two variants, L1156F and R1453W, are unique to Japanese patients $^{12-16,24}$ and $\mathrm{Q} 1352 \mathrm{H}$ is known to be unique in Asian patients ${ }^{13,14,16,24}$. These reports were all in the Japanese or Asian samples, and data from 1000 Genomes studies suggest no variants are found in other ethnicities.

In this study, eight non-synonymous variants, R31C, E217G, R352Q, V470M, I556V, L1156F, Q1352H and R1453W, were found in our patients. Nine out of 28 patients had a V470M variant (Table 2). It is known that channel activity of V470 CFTR protein is lower than that of M470 CFTR ${ }^{23,24}$. Cuppens et al. ${ }^{23}$ suggested that M470 CFTR proteins matured more slowly than V470 CFTR, although M470 CFTR had higher channel activity than 


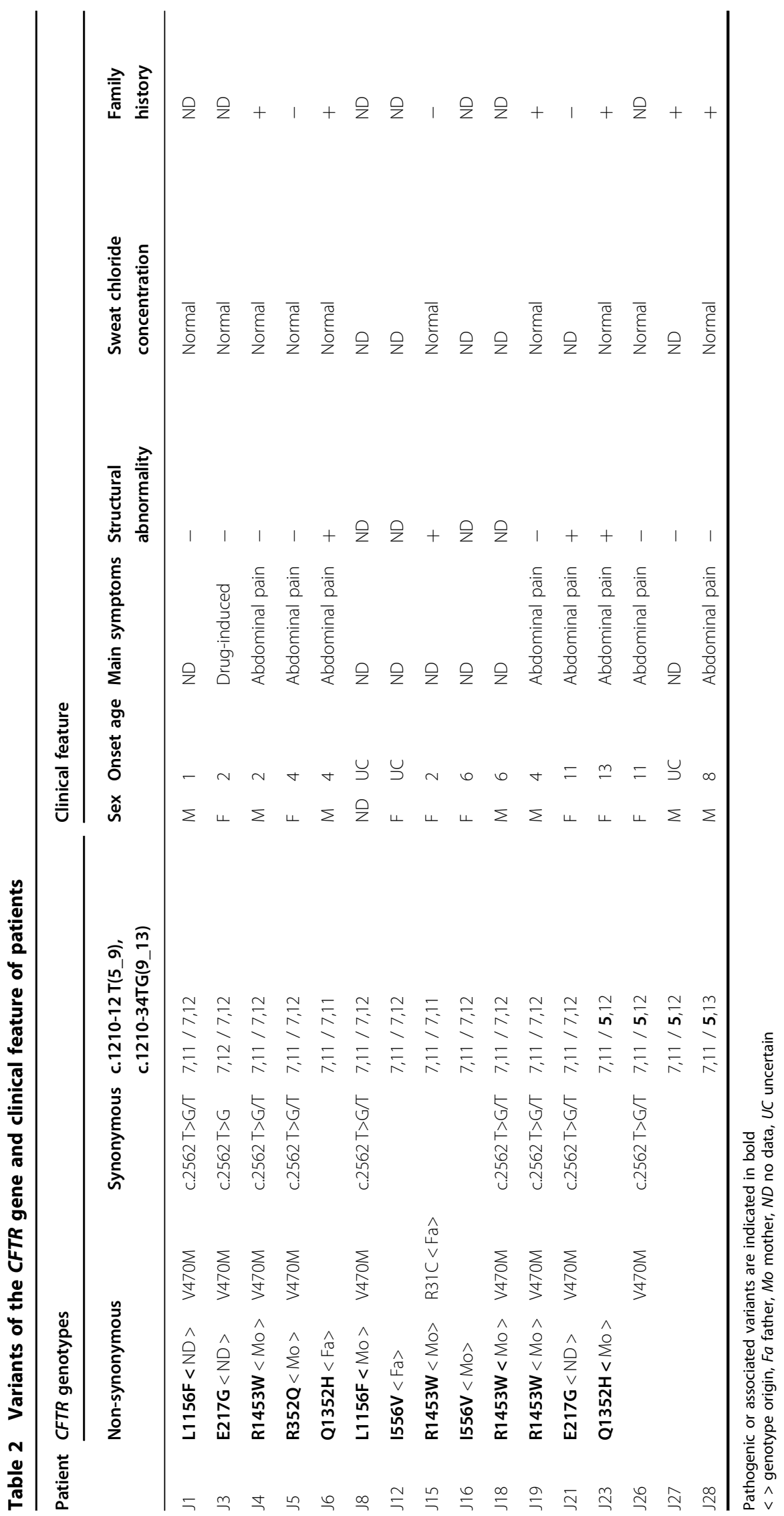


V470 CFTR. Hence, the proteins perform complementary functions to each other, suggesting that the variant is benign. However, these studies displayed that the V470M locus affected the penetrance of another variants' expression, such as c.1210-12 $\mathrm{T}(5)^{14,24}$. There are many reports about the contribution of the V470M locus to other variants ${ }^{13-16,23,24}$ and it is possible that the V470M locus affects the penetrance of other functionally affected variants, such as E217G, R352Q, L1156F, and R1453W. However, more experiments are needed to confirm these findings.

For the gene variants R31C, E217G, R352Q, I556V, L1156F, Q1352H and R1453W, there are reports that they affect protein activity and/or expression ${ }^{14,24,27}$. Lee et al. ${ }^{14}$ reported that the variants, E217G, I556V, Q1352H and R1453W of CFTR decreased channel activity, which implies an association with pancreatitis in Japanese patients. The L1156F CFTR, which is associated with alcoholic chronic pancreatitis in the Japanese, causes impaired CFTR function and expression in combination with the V470M variant ${ }^{24}$. This variant might also affect paediatric pancreatitis.

The $\mathrm{R} 352 \mathrm{Q}$ and $\mathrm{R} 1453 \mathrm{~W}$ variants were significantly more frequent in patients (Table 1). According to the Exome Aggregation Consortium, the R352Q variant is rare in Caucasian population with an incidence of 6 in $121,412(0.0002 \%)^{28}$. The R352 CFTR is a residue flanking the predicted cytoplasmic end of the M6 segment (Fig. 2) and the Q352 leads to a decrease in anion-selective activity in the channel ${ }^{29}$. The R1453W variant is not found in either Chinese and Korean patients with idiopathic pancreatitis ${ }^{12,14}$. The W1453 CFTR variant also affects the protein function and gene expression ${ }^{14}$. In the previous study, two patients out of 128 patients had a heterozygous variant of p.A137G in CPA $1^{17}$. Of the two patients, one patient had also had the p.R1453W variant in CFTR, suggesting that the patient was affected not only by CPA1 A137G but also CFTR R1453W.

We evaluated the CFTR gene expression in patient J15, who had two heterozygote variants, R31C and R1453W. Jurkuvenaite et $\mathrm{al}^{27}$ reported that $\mathrm{R} 31 \mathrm{C}$ leads to decreased expression at the cell surface and diminished $\mathrm{Cl}^{-}$channel activity. The expression analysis showed that neither variant affected CFTR gene expression and splicing in our patient. Lee et al. ${ }^{14}$ reported that R1453WCFTR showed mild reduction of open probability. Thus, both variants might be associated with the development of pancreatitis after translation.

A splice-affecting variant, $5 \mathrm{~T}$ in intron 9 was identified in four patients. We detected exon 10 skipping of CFTR in J28 and his mother in the nasal swab cells (Fig. 1). Both had long TG repeats (13TG) adjacent to 5T, which confirms the previous study results that longer TG repeats increase risk of exon 10 skipping $^{30}$. The transcripts completely skipped exon 10, whereas the patient had 11TG repeat and 7T in another allele. Rave-Harel et al. ${ }^{31}$ reported that the degree of exon 10 skipping with variant $5 \mathrm{~T}$ was variable and penetrance of disease expression was also different in individuals. Although patient J15 and the non-affected father of J28 have the same genotype, (TG) 11/11 and (T) 7/7, partial skipping of CFTR exon 10 occurred only in patient $\mathrm{J} 15$, suggesting that other genetic factors might affect splicing ${ }^{31}$.

The skipping of exon 10 causes the deletion of first $21 \%$ of nucleotide-binding domain 1 where the common $\mathrm{CF}$ mutation $\Delta 508$ is found in Caucasians ${ }^{5,32}$. The domain

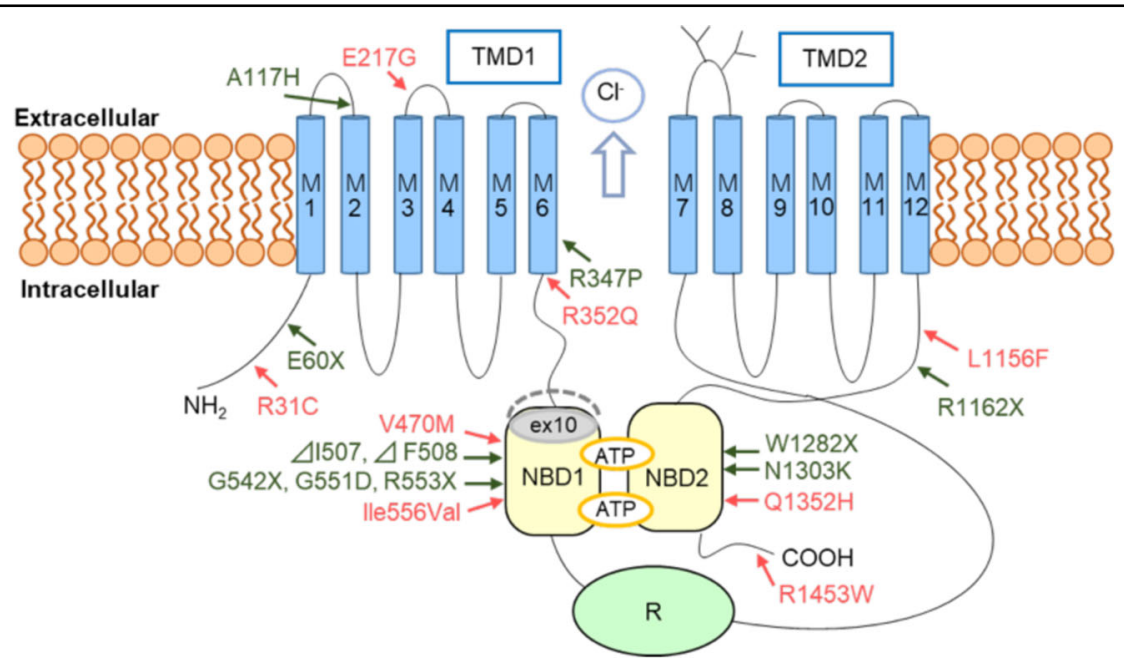

Fig. 2 Schematic diagram of the predicted protein structure of CFTR and locations of variants. The non-synonymous variants, which were found in this study, indicated as arrows in red colour. The common non-synonymous variants in Caucasian were indicated as arrows in green colour. The amino acids encoded in exon 10 (within the NBD1 domain) were indicated as shaded areas (grey) 
seems to be a critical region associated to diseases. Patient J28 and his mother had no symptoms of respiratory involvement and only of pancreatitis. This suggests that exon 10 skipping might affect pancreatitis more so rather CF.

We found many intronic variants in the patients with paediatric pancreatitis, which were unique or significantly more frequent compared with the Japanese population (Supplementary Tables S4, 5). According to the association study, it was reported that some synonymous and/or non-exonic variants are involved in pancreatitis ${ }^{24}$. Therefore, it is possible that some variants are involved in genetic risk factors for paediatric pancreatitis. However, further experiments should be needed to evaluate the relationships between intronic variants and pancreatitis.

In the present study, the frequency of pathogenic and functionally affected CFTR variants in Japanese paediatric patients with pancreatitis was $1 / 28$ (3.6\%) and 15/28 (53.6\%), respectively. However, as patients in this study were not found to have pathogenic variants in the causative genes for pancreatitis in a previous study, the actual frequency in pancreatitis is likely lower. Taken together with our previous study ${ }^{17}$, the frequency of having such pathogenic or related variant of CFTR is at least more than $12.5 \%(16 / 128)$. As the CFTR gene is not causative but rather an associate gene for pancreatitis, pathogenic variants may affect developing pancreatitis ${ }^{33}$. The CFTR pathogenic or functionally affected variants found in this study may affect pancreatitis in Japanese populations as well.

In summary, we found that one out of 28 (3.6\%) and 15 out of 28 (53.6\%) Japanese patients with paediatric pancreatitis had a pathogenic and functionally affected variant in CFTR, respectively. As the 28 patients studied were not diagnosed after genetic analysis of known causative genes for pancreatitis in a previous study ${ }^{17}$, the actual frequency of CFTR variants in Japanese paediatric pancreatitis will be lower than demonstrated in this study. Considering results of the present and previous studies, this suggests that the frequency of functionally affected variants of CFTR is estimated to be at least $12.5 \%$. Variants of the CFTR gene in the Japanese were previously thought to be rare, as CF is a very rare disease estimated at $1 / 350,000$ in $\operatorname{Japan}^{25}$. However, this study suggests that pathogenic or functionally affected variants of CFTR might not be so rare in Japanese paediatric patients with idiopathic pancreatitis. Thus, CFTR may also present as a genetic risk factor in paediatric pancreatitis in Japanese. Surveying variants of the CFTR gene might help determine risk of pancreatitis in Japanese children.

\section{Acknowledgements}

We thank all participating patients and their families. We also thank Riko Kaizuka (Department of Pediatrics, Hirosaki Municipal Hospital) and Jun
Murakami (Division of Pediatrics and Perinatology, Faculty of Medicine, Tottori University) for collaborating with the data collection.

\section{Funding}

This study was supported in part by a Grant-in-Aid from the Ministry of Health, Labour and Welfare, Japan (H27-Shippei-Taisaku (Kaken) 20145117A; T.S.), and by a Grant of Practical Research Project for Rare/Intractable Diseases from Japan Agency for Medical Research and Development, Japan (18ek0109234h0002; T.K.).

\section{Conflict of interest}

The authors declare that they have no conflict of interest.

\section{Publisher's note}

Springer Nature remains neutral with regard to jurisdictional claims in published maps and institutional affiliations.

Supplementary Information is available for this paper at https://doi.org/ 10.1038/s41439-019-0049-7.

Received: 9 December 2018 Revised: 15 February 2019 Accepted: 18 February 2019.

Published online: 11 April 2019

\section{References}

1. Whitcomb, D. C. et al. Hereditary pancreatitis is caused by a mutation in the cationic trypsinogen gene. Nat. Genet. 14, 141-145 (1996).

2. Witt, $\mathrm{H}$. et al. Mutations in the gene encoding the serine protease inhibitor, Kazal type 1 are associated with chronic pancreatitis. Nat. Genet. 25, 213-216 (2000).

3. Rosendahl, J. et al. Chymotrypsin C (CTRC) variants that diminish activity or secretion are associated with chronic pancreatitis. Nat. Genet. 40, 78-82 (2008).

4. Witt, $\mathrm{H}$. et al. Variants in CPA1 are strongly associated with early onset chronic pancreatitis. Nat. Genet. 45, 1216-1220 (2013).

5. Riordan, J. R. et al. Identification of the cystic fibrosis gene: cloning and characterization of complementary DNA. Science 245, 1066-1073 (1989).

6. Cohn, J. A. et al. Relation between mutations of the cystic fibrosis gene and idiopathic pancreatitis. N. Engl. J. Med. 339, 653-658 (1998).

7. Sharer, N. et al. Mutations of the cystic fibrosis gene in patients with chronic pancreatitis. N. Engl. J. Med. 339, 645-652 (1998).

8. Cutting, G. R. Cystic fibrosis genetics: from molecular understanding to clinical application. Nat. Rev. Genet. 16, 45-56 (2015).

9. Johns Hopkins University. CFTR2 (2011). https://www.cftr2. org. Accessed 4 November 2018.

10. Masson, E., Chen, J. M., Audrézet, M. P., Cooper, D. N. \& Férec, C. A conservative assessment of the major genetic causes of idiopathic chronic pancreatitis: data from a comprehensive analysis of PRSS1, SPINK1, CTRC and CFIR Genes in 253 young French patients. PLOS ONE 8, e73522 (2013).

11. Rosendahl, J. et al. CFTR, SPINK1, CTRC and PRSS1 variants in chronic pancreatitis: is the role of mutated CFIR overestimated? Gut 62, 582-592 (2013).

12. Xiao, Y. et al. Targeted gene next-generation sequencing in Chinese children with chronic pancreatitis and acute recurrent pancreatitis. J. Pediatr. 191, 158-163 (2017).

13. Nakano, E. et al. Targeted next-generation sequencing effectively analyzed the cystic fibrosis transmembrane conductance regulator gene in pancreatitis. Dig. Dis. Sci. 60, 1297-1307 (2015).

14. Lee, J. H. et al. A haplotype-based molecular analysis of CFTR mutations associated with respiratory and pancreatic diseases. Hum. Mol. Genet. 12, 2321-2332 (2003)

15. Fujiki, K. et al. Genetic evidence for CFTR dysfunction in Japanese: background for chronic pancreatitis. J. Med. Genet. 41, 55-61 (2004).

16. Chang, M. C. et al. Spectrum of mutations and variants/haplotypes of CFTR and genotype-phenotype correlation in idiopathic chronic pancreatitis and controls in Chinese by complete analysis. Clin. Genet. 71, 530-539 (2007). 
17. Saito, N. et al. Genetic analysis of Japanese children with acute recurrent and chronic pancreatitis. J. Pediatr. Gastroenterol. Nutr. 63, 431-436 (2016).

18. Broad Institute and the Regents of the University of California. Integrative Genomics Viewer (IGV) (2013). http://software.broadinstitute.org/software/igv. Accessed 4 November 2018.

19. Ramalho, A. S. et al. Methods for RNA extraction, CDNA preparation and analysis of CFTR transcripts. J. Cyst. Fibros. 3, 11-15 (2004).

20. Bronckers, A. et al. The cystic fibrosis transmembrane conductance regulator (CFTR) is expressed in maturation stage ameloblasts, odontoblasts and bone cells. Bone 46, 1188-1196 (2010).

21. Tohoku Medical Megabank Organization. Integrative Japanese Genome Variation (iJGVD). https://ijgvd.megabank.tohoku.ac.jp. Accessed 4 November 2018.

22. 1000 Genomes Project Consortium. International Genome Sample Resource (IGSR). http://www.internationalgenome.org. Accessed 4 November 2018.

23. Cuppens, $H$. et al. Polyvariant mutant cystic fibrosis transmembrane conductance regulator genes. The polymorphic (TG)m locus explains the partial penetrance of the T5 polymorphism as a disease mutation. J. Clin. Invest. 101, 487-496 (1998).

24. Kondo, S. et al. Functional characteristics of L1156F-CFTR associated with alcoholic chronic pancreatitis in Japanese. Am. J. Physiol. Gastrointest. Liver Physiol. 309, 260-269 (2015).

25. Yamashiro, Y. et al. The estimated incidence of cystic fibrosis in Japan. J. Pediatr. Gastroenterol. Nutr. 24, 544-547 (1997).
26. Singh, M., Rebordosa, C., Bernholz, J. \& Sharma, N. Epidemiology and genetics of cystic fibrosis in Asia: In preparation for the next-generation treatments. Respirology 20, 1172-1181 (2015).

27. Jurkuvenaite, A. et al. Mutations in the amino terminus of the cystic fibrosis transmembrane conductance regulator enhance endocytosis. J. Biol. Chem. 281, 3329-3333 (2006).

28. Exome Aggregation Consortium. ExAC Brower Beta (2014). http://exac. broadinstitute.org. Accessed 4 November 2018.

29. Guinamard, R. \& Akabas, M. H. Arg352 is a major determinant of charge selectivity in the cystic fibrosis transmembrane conductance regulator chloride channel. Biochemistry 38, 5528-5537 (1999).

30. Groman, J. D. et al. Variation in a repeat sequence determines whether a common variant of the cystic fibrosis transmembrane conductance regulator gene is pathogenic or benign. Am. J. Hum. Genet. 74, 176-179 (2004).

31. Rave-Harel, N. et al. The molecular basis of partial penetrance of splicing mutations in cystic fibrosis. Am. J. Hum. Genet. 60, 87-94 (1997).

32. Chu, C. S., Trapnell, B. C., Curristin, S., Cutting, G. R. \& Crystal, R. G. Genetic basis of variable exon 9 skipping in cystic fibrosis transmembrane conductance regulator mRNA. Nat. Genet. 3, 151-156 (1993).

33. Teich, N., Ockenga, J., Keim, V. \& Mössner, J. Genetic risk factors in chronic pancreatitis. J. Gastroenterol. 37, 1-9 (2002).

34. Vankeerberghen, A., Cuppens, H. \& Cassiman, J.J. The cystic fibrosis transmembrane conductance regulator: an intriguing protein with pleiotropic functions. J. Cyst Fibros. 1, 13-29 (2002). 